\title{
A yeast three-hybrid system that reconstitutes mammalian hypoxia inducible factor regulatory machinery
}

\author{
Maria L Alcaide-German ${ }^{1}$, Alicia Vara-Vega ${ }^{2}$, Luis F Garcia-Fernandez ${ }^{3}$, \\ Manuel O Landazuri² and Luis del Peso*1
}

Address: 'Departamento de Bioquímica, Instituto de Investigaciones Biomédicas 'Alberto Sols', Consejo Superior de Investigaciones Científicas, Universidad Autónoma de Madrid, Arturo Duperier 4, 28029 Madrid, Spain, ²Servicio de Inmunología, Hospital de la Princesa-UAM, Diego de León, 62. 28006 Madrid, Spain and 3R\&D Department, PharmaMar, S.A.PharmaMar, Avenida de los reyes, 1. E-28770 Colmenar Viejo, Spain

Email: Maria L Alcaide-German - malcaide@iib.uam.es; Alicia Vara-Vega - avara.hlpr@salud.madrid.org; Luis F Garcia-

Fernandez - lfgarcia@pharmamar.com; Manuel O Landazuri - mortiz.hlpr@salud.madrid.org; Luis del Peso* - lpeso@iib.uam.es

* Corresponding author

Published: 10 April 2008

BMC Cell Biology 2008, 9:18 doi:10.1/86/147|-2121-9-18

This article is available from: http://www.biomedcentral.com/l47/-2/2I/9//8

(C) 2008 Alcaide-German et al; licensee BioMed Central Ltd.

This is an Open Access article distributed under the terms of the Creative Commons Attribution License (http://creativecommons.org/licenses/by/2.0), which permits unrestricted use, distribution, and reproduction in any medium, provided the original work is properly cited.
Received: 17 January 2008

Accepted: 10 April 2008

\begin{abstract}
Background: Several human pathologies, including neoplasia and ischemic cardiovascular diseases, course with an unbalance between oxygen supply and demand (hypoxia). Cells within hypoxic regions respond with the induction of a specific genetic program, under the control of the Hypoxia Inducible Factor (HIF), that mediates their adaptation to the lack of oxygen. The activity of HIF is mainly regulated by the EGL-nine homolog (EGLN) enzymes that hydroxylate the alpha subunit of this transcription factor in an oxygen-dependent reaction. Hydroxylated HIF is then recognized and ubiquitinilated by the product of the tumor suppressor gene, PVHL, leading to its proteosomal degradation. Under hypoxia, the hydroxylation of HIF by the EGLNs is compromised due to the lack of oxygen, which is a reaction cosubstrate. Thus, HIF escapes degradation and drives the transcription of its target genes. Since the progression of the aforementioned pathologies might be influenced by activation of HIF-target genes, development of small molecules with the ability to interfere with the HIF-regulatory machinery is of great interest.
\end{abstract}

Results: Herein we describe a yeast three-hybrid system that reconstitutes mammalian HIF regulation by the EGLNs and VHL. In this system, yeast growth, under specific nutrient restrictions, is driven by the interaction between the $\beta$ domain of $\mathrm{VHL}$ and a hydroxyproline-containing HIF $\alpha$ peptide. In turn, this interaction is strictly dependent on EGLN activity that hydroxylates the HIF $\alpha$ peptide. Importantly, this system accurately preserves the specificity of the hydroxylation reaction toward specific substrates. We propose that this system, in combination with a matched control, can be used as a simple and inexpensive assay to identify molecules that specifically modulate EGLN activity. As a proof of principle we show that two known EGLN inhibitors, dimethyloxaloylglycine (DMOG) and 6-chlor-3-hydroxychinolin-2-carbonic acid-N-carboxymethylamide (S9567II), have a profound and specific effect on the yeast HIF/EGLN/VHL system.

Conclusion: The system described in this work accurately reconstitutes HIF regulation while preserving EGLN substrate specificity. Thus, it is a valuable tool to study HIF regulation, and particularly EGLN biochemistry, in a cellular context. In addition, we demonstrate that this system can be used to identify specific inhibitors of the EGLN enzymes. 


\section{Background}

Most mammalian tissues are strictly dependent on oxidative metabolism and require constant oxygen supply to maintain cell function. Thus, acute oxygen deprivation, even for brief periods of time, can be deleterious. In contrast, cells can adapt to moderate chronic hypoxia through the induction of a specific gene expression program. The induction of this set of genes adjusts the metabolism by increasing anaerobic glycolysis [1] and fine-tuning mitochondrial respiration [2,3]. In addition, hypoxic cells induce vascularization of the poorly oxygenated tissue through the expression of proangiogenic molecules such as VEGF [4]. The family of Hypoxia Inducible Factors (HIFs) is responsible for the induction of the vast majority of the genes involved in these adaptive responses $[5,6]$. HIF transcription factors are heterodimers of an oxygen dependent alpha subunit (HIF $\alpha$ ) and a beta (HIF $\beta$ ) chain that is shared with other transcription factors of the basichelix-loop-helix family. There are three different HIF $\alpha$ isoforms (HIF $1 \alpha$, HIF $2 \alpha$ and HIF $3 \alpha$ ), encoded by independent genes, whose regulation by oxygen is thought to be similar. Oxygen affects the half life $[7,8]$ as well as the transcriptional activity [9] of HIF $\alpha$ proteins. In both cases, the effect is mediated by hydroxylation of specific residues, within HIF $\alpha$, by a family of 2-oxogluatarate-dependent dioxygenases that require molecular oxygen as cosubstrate. These modifications affect the ability of HIF to interact with other proteins. Specifically, hydroxylation of a conserved asparagine residue (N803 in human HIF1 $\alpha$ protein) within the $\mathrm{C}$-terminal transactivation domain by the Factor Inhibiting HIF (FIH) $[10,11]$ prevents its interaction with the p300 coactivator [9]. On the other hand, the activity of the EGL-nine homologues (EGLNs, [12]) results in the hydroxylation of two conserved proline residues (P402 and P564 in human HIF1 $\alpha$ ) within the Oxygen-Dependent Degradation domain (ODD) of HIF $\alpha$ proteins[13]. Proline hydroxylation allows the binding of the E3 ubiquitin ligase pVHL to HIF $\alpha$ leading to its ubiquitination and proteosomal degradation $[8,14,15]$. Since FIH and EGLNs require oxygen as cosubstrate, under hypoxia their activity is reduced $[16,17]$. As a consequence the interaction with pVHL is lost, leading to HIF stabilization, while the interaction with p300 is allowed resulting in enhanced transcriptional activity. Although the regulation of HIF transcriptional activity seems to be critical for the induction of some HIF-target genes during hypoxia $[18]$, the major effect of oxygen is the regulation of HIF $\alpha$ stability through the activity of the EGLNs. To date three independent enzymes termed EGLN1, 2 and 3 (also known as PHD2, 1 and 3 respectively) have been described to mediate HIF $\alpha$ proline hydroxylation $[7,19,20]$. Intriguingly, these enzymes display substrate specificity: while EGLN1 and 2 are capable of hydroxylating both HIFa prolines, EGLN3 is unable to act upon the N-terminal residue (P402 in human HIF1 $\alpha$ ) [17,21-23].
In this regard, recent publications describe novel potential targets for these enzymes that seem to be regulated by specific isoforms $[24,25]$.

In addition to the relevance of this pathway in the physiological response to reduced oxygen supply, it probably plays a major role during the progression of several pathologies that course with hypoxia. Specifically, activation of HIF has been implicated in the induction of the glycolitic and angiogenic phenotype of cancer [4,26], generation and progression of tumors [27] and resistance to the isquemic insults (preconditioning) [28,29] among other phenomena. Thus, the identification of small molecules that modulate this pathway is of great therapeutical interest. In fact, several molecules with the ability to modulate HIF activity have been already described. In animal models, HIF inhibitors have been proven useful in the treatment of tumors [30], while molecules with the ability to induce HIF result in angiogenesis in vivo [31].

In this work, we describe the generation of a three-hybrid system that accurately reconstitutes mammalian HIF $\alpha$ regulation by EGLN hydroxylation and subsequent VHL binding. This system can be of aid in the study of EGLN biochemistry and the characterization of novel substrates. In addition, we demonstrate that it can be used as an efficient assay to screen for small molecules with the ability to regulate HIF.

\section{Results \\ Generation of a yeast three hybrid-based system that reconstitutes HIF regulation}

Several EGLN assays have been described which include the determination of enzyme activity by measure of cosubstrates (dioxygen and 2-oxoglutarate) consumption/ $\mathrm{CO}_{2}$ production and the detection of HIF hydroxylation using mass spectrometry or capture of VHL $[32,33]$. In addition, strategies to determine EGLN activity within cells have also been developed. These include the detection of reporter proteins fused to ODD [34] and the use of antibodies raised against hydroxyproline-containing ODDs [35]. The major limitation of the in vitro assays is that they are performed in a non physiological environment. On the other hand, the cell-based assays have to deal with the endogenous HIF machinery. To circumvent these problems we sought to reconstitute the HIF regulatory machinery in yeast cells, where expressed proteins are in a cellular environment but where no endogenous proteins related to the HIF pathway are expressed, therefore eliminating possible sources of interference. To this end, we cloned a fragment of human HIF1 $\alpha$ (residues 554576) containing P564 in frame with yeast Gal4 activation domain (AD-P564) (figure 1A). This fragment is sufficient for EGLN recognition [23] and VHL binding [36]. In addition, we cloned a VHL fragment encoding for its $\beta$-domain 
A
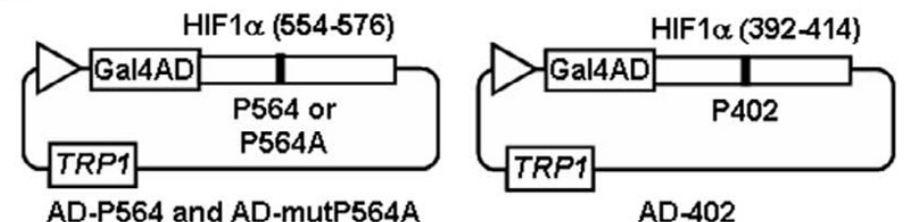

AD-402

VHL (63-157)

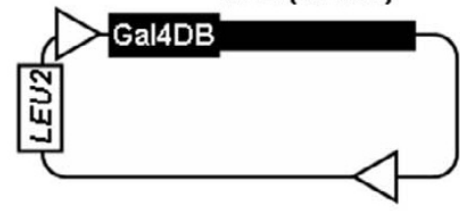

DB-VHL

C

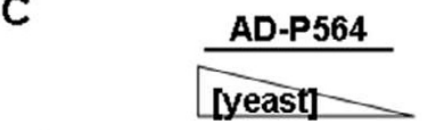

DB-VHL

E3/DB-VHL

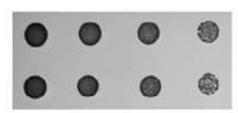

DB-VHL

E3/DB-VHL

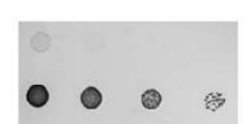

DB-VHL

E3/DB-VHL

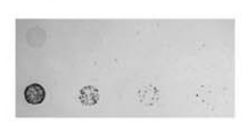

VHL (63-157)

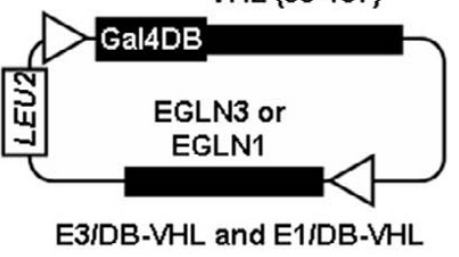

D

AD-P564

AD-P402

AD-mutP564A

INTERACTION (medium str.)

INTERACTION

AD-P564

AD-P402

AD-mutP564A

AD-P564 (high str.)

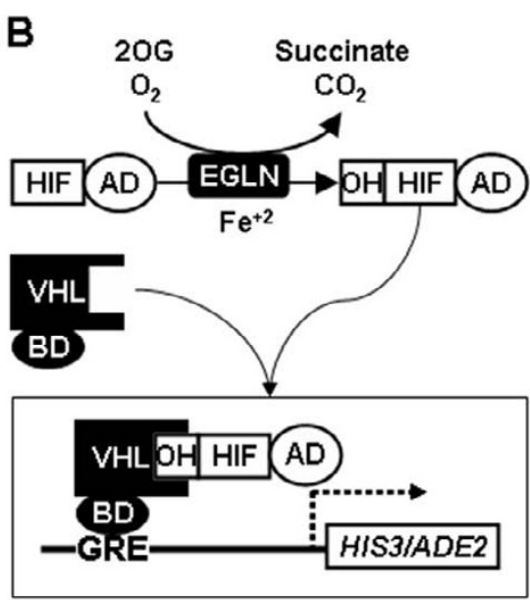

E3/DB-VHL E1/DB-VHL
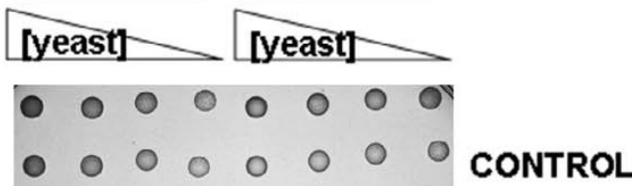

AD-P402

AD-mutP564A

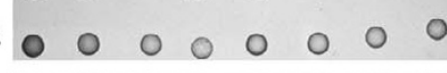

CONTROL

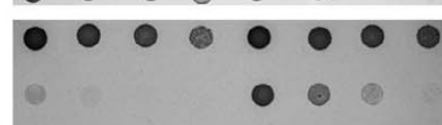

INTERACTION (medium str.)

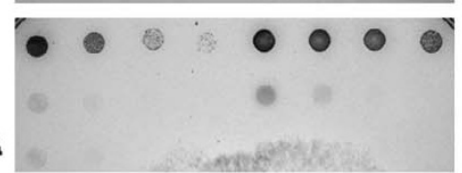

INTERACTION (high str.)

\section{Figure I}

Characterization of a yeast three-hybrid system that reconstitutes HIF regulatory machinery. A, Schematic diagram representing the yeast three-hybrid constructs. Numbers in brackets correspond to residue positions for the encoded proteins. TRPI and LEU2, genes encoding for the enzymes that allow cell growth in the absence of Trp and Leu respectively. B, Schematic diagram of the interaction between the different system components. $20 \mathrm{G}, 2$-oxoglutarate. $C$ and $D$, Serial dilutions of clones transformed with the indicated constructs were grown on plates lacking Leu and Trp (CONTROL), plates lacking Leu, Trp and His (INTERACTION, medium str.) or plates lacking Leu, Trp, His and adenine (INTERACTION, high str.). The results shown are representative of at least three independent experiments.

(residues 63 to 157) in frame with the yeast Gal4 DNA binding domain (DB-VHL) (figure 1A). The VHL $\beta$ domain is involved in HIF1 $\alpha$ binding, while the $\alpha$ domain, missing in this construct, interacts with the elongins $\mathrm{B} / \mathrm{C}$ that are required to promote HIF $\alpha$ ubiquitination. Then we tested the interaction between these fusion proteins as their ability to support the growth of a yeast strain conditionally auxotrophic for histidine and adenine (figure $1 \mathrm{~B}$ ). As shown in figure $1 \mathrm{C}$, yeast cells transformed with AD-P564 in combination with DB-VHL failed to grow in plates lacking histidine, suggesting lack of significant interaction between the two fusion proteins.
This result was expected since no EGLN orthologs are present in yeast and VHL binding to HIF1 $\alpha$ is strictly dependent on proline hydroxylation $[8,14]$. In addition, these data indicates that residual binding of VHL to nonhydroxylated AD-P564 is below detection limit in our assay conditions. Importantly, the expression of EGLN3 (construct E3/DB-VHL, figure 1A), together with the two fusion proteins, is sufficient to promote direct binding of DB-VHL to AD-P564, as indicated by the growth of transformed yeast in restrictive plates (figure $1 \mathrm{C}$ ). The effect was not restricted to EGLN3 since the expression of EGLN1 also triggered the interaction between the fusion 
proteins (figure 1D). To further confirm that DB-VHL/ADP564 interaction was mediated by the hydroxylation of AD-P564 by EGLNs, we repeated the experiment using a mutant HIF peptide in which P564 was replaced by an alanine residue (AD-mutP564A, figure 1A). As shown in figure $1 \mathrm{D}, \mathrm{DB}-\mathrm{VHL}$ was unable to interact with $\mathrm{AD}$ mutP564A regardless of the presence of either EGLN. Moreover, in spite of relaxed target requirements [23,37], it has been described that EGLN isoforms display a specific preference for the two target sequences in HIF1 $\alpha$ [17,21-23]. Therefore, we next wanted to determine whether in this system the EGLNs retained their native substrate specificity. Figure 1D shows that EGLN3 efficiently hydroxylates the peptide containing the sequence surrounding proline 564 (AD-P564), but it has no detectable activity toward a peptide comprising residues 392414 derived from HIF1 $\alpha$ (AD-P402). In contrast, EGLN1 was able to hydroxylate both sequences allowing their binding to VHL (figure 1D). Interestingly, the activity of EGLN1 toward AD-P402 was slightly lower than its activity using AD-P564 substrate as evidenced by the different growth of yeast transformed with each sequence in the high stringency plates (figure 1D). These results are in perfect agreement with previous reports describing the activity of the EGLN isoforms against different substrates [23,37-39]. Finally, further confirmation that EGLNs retained their substrate specificity was obtained through the analysis of the effect of L574A and L562R mutations (Additional file 1).

All together, these results indicate that this system faithfully reconstitutes the regulation of HIF $\alpha$ proteins by the sequential action of EGLN enzymes and VHL binding. In addition, data show that the $\beta$-domain of VHL is sufficient for target recognition and binding.

\section{The reconstituted HIF/EGLN/VHL system can be exploited to identify specific EGLN inhibitors}

The system described above relies on EGLN activity for DB-VHL/AD-P564 interaction, thus we reasoned that EGLN inhibitors should interfere with yeast growth. However, in cell-based assays, the inhibitory effects of drugs have to be carefully controlled for unspecific side effects on cell physiology or on the assay system itself. In order to have a control for the drug specificity and rule out potential side effects on yeast growth, we generated a set of constructs analogous to those described above but based on a protein machinery unrelated to the HIF pathway. In this control system, yeast growth is dependent on the interaction between an immunoreceptor tyrosine-based activation motif (ITAM), derived from the type I $\gamma$ IgE receptor (FceRI $\gamma$ ), and the Src-Homology domain 2 (SH2) from the Syk protein. Importantly, the binding of Syk-SH2 tandem domains to the FceRI $\gamma$-ITAM is dependent on the tyrosine phosphorylation of the latter [40]. The vectors generated for this system (figure 2A) encode for a Sykderived tandem $\mathrm{SH} 2$ domains fused to the activation domain of Gal4 (AD-SH2) and for an ITAM from FceRI $\gamma$ fused to the Gal4 DNA binding domain (DB-ITAM). Finally, the cytoplasmic tyrosine kinase Lck was cloned into the pBridge vector to induce DB-ITAM phosphorylation and trigger its binding to $\mathrm{AD}-\mathrm{SH} 2$ (figure $2 \mathrm{~B}$ ). Since yeast do not contain orthologues of the proteins of this system, AD-SH2 did not significantly interact with DBITAM unless the tyrosine kinase Lck was co-expressed in the assay (figure 2C), as previously reported [40]. Thus, the Lck and EGLN-based assays are analogous in that both have three components: an enzyme and two fusion proteins whose interaction allows yeast growth in restrictive media. Additionally, in both systems the interaction between the fusion proteins, and thus yeast growth, requires a modification of one of these proteins by the enzyme (compare figures $1 \mathrm{~B}$ and $2 \mathrm{~B}$ ).

Next we tested the ability of these combined systems to detect molecules that interfere with HIF regulation. For this purpose we selected two previously characterized EGLN inhibitors, dimethyloxaloylglycine (DMOG) [41] and 6-chlor-3-hydroxychinolin-2-carbonic acid-N-carboxymethyl-amide (S956711) [31]. In order to facilitate the quantification of the effects of inhibitors on these systems, we set up the experiments in liquid media so that yeast growth could readily be determined by the optical density of the cell culture. Since the growth rate of yeast transformed with different constructs in each culture media varies, we adjusted the initial concentration of each yeast strain for each media so that all cultures achieved logarithmic growth in overlapping time windows (Additional file 2). As shown in figure $3 \mathrm{~A}$, treatment with increasing doses of $S 956711$ had a strong effect on the growth of yeast expressing AD-HIF and DB-VHL/EGLN3 in restrictive media. In contrast, when these cells were cultured in control media, where no interaction between fusion proteins was required for growth, S956711 had only a minor effect when used at $100 \mu \mathrm{M}$ (figure 3A). Thus, the effect of S956711 is not due to an unspecific effect on yeast viability/growth. To further confirm the specificity of the effect upon the HIF system, we tested the effect of S956711 on yeast transformed with AD-SH2 and DB-ITAM/Lck. As shown in figure 3, S956711 had no significant effect in this control system regardless of the culture media. These results rule out that the inhibition of the EGLN system could be due to side effects of S956711 on transcription/translation of the three-hybrid elements. The specific inhibitory effect of S956711 was highly reproducible and unspecific effects were undetectable at doses below $100 \mu \mathrm{M}$ (figure 3B). Similarly to the results observed for S956711, treatment with DMOG also resulted in a dose-dependent inhibition of the interaction between AD-HIF and DB-VHL (figure 4A). As shown for 
A

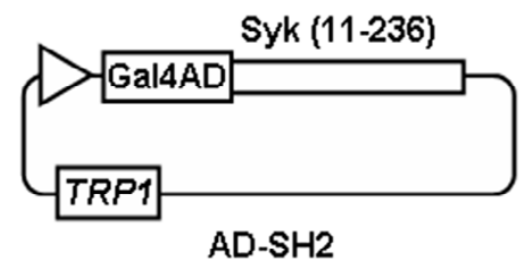

FceRly (45-86)

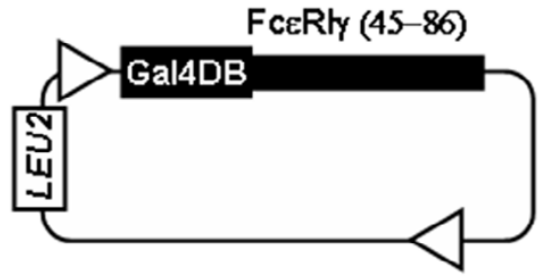

DB-ITAM

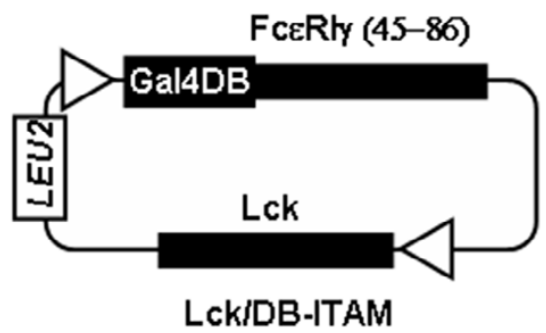

B

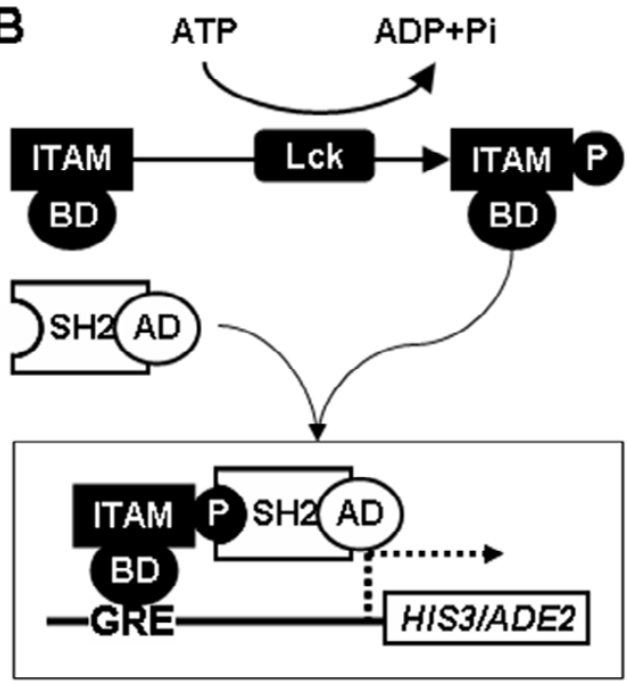

C
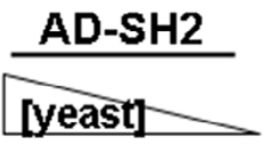

DB-ITAM

Lck/DB-ITAM

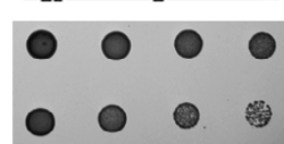

CONTROL

DB-ITAM

Lck/DB-ITAM

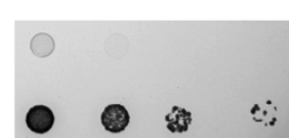

INTERACTION (medium str.)

DB-ITAM

Lck/DB-ITAM
INTERACTION

(high str.)

Figure 2

Characterization of a control yeast three-hybrid system based on an ITAM/SH2 interaction that depends on

Lck activity. A, Schematic diagram representing the constructs. Abbreviations are as in figure I. B, Schematic diagram of the interaction between the different system components. C, Serial dilutions of clones transformed with the indicated constructs were grown on different astringency plates. Symbols, abbreviations and panel labels are as in figure I. The results shown are representative of at least three independent experiments.

S956711, DMOG inhibition was specific for the HIF regulatory machinery, since no effect was observed on the binding of AD-SH2 to DB-ITAM (figures $4 \mathrm{~A}$ and $4 \mathrm{~B}$ ).

DMOG is a 2-oxoglutarate analogue that probably inhibits EGLN activity by competition with the reaction cosubstrate 2-oxoglutarate [42]. Thus, DMOG should affect EGLN catalitic activity (substrate hydroxylation), but not substrate binding. With the purpose of testing this hypothesis, we investigated the effect of DMOG on EGLN3 binding to HIF using a two hybrid-based assay [23]. In this assay, yeast growth in restrictive media is dependent on the direct interaction between a Gal-4 DNA binding domain-EGLN3 (DB-E3) and AD-HIF fusion proteins (figures $5 \mathrm{~A}$ and $5 \mathrm{~B}$ ). The interaction between DB-E3 and AD-HIF is specific since a single mutation on the target proline in HIF sequence abolishes yeast growth (figure 5C) [23]. Importantly, this assay allows the direct comparison of DMOG effect on EGLN3 ability to bind HIF and act upon it. As shown in figure 5D, DMOG treatment had no effect on the direct binding of EGLN3 to its substrate, as demonstrated by the lack of effect on the growth of yeast expressing DB-EGLN3 and AD-HIF (E3/HIF system). However, at the same doses it clearly affected the hydroxylation of AD-HIF by EGLN3 (figure 5D, VHL/HIF system). This result indicates that, as expected, DMOG does 
A
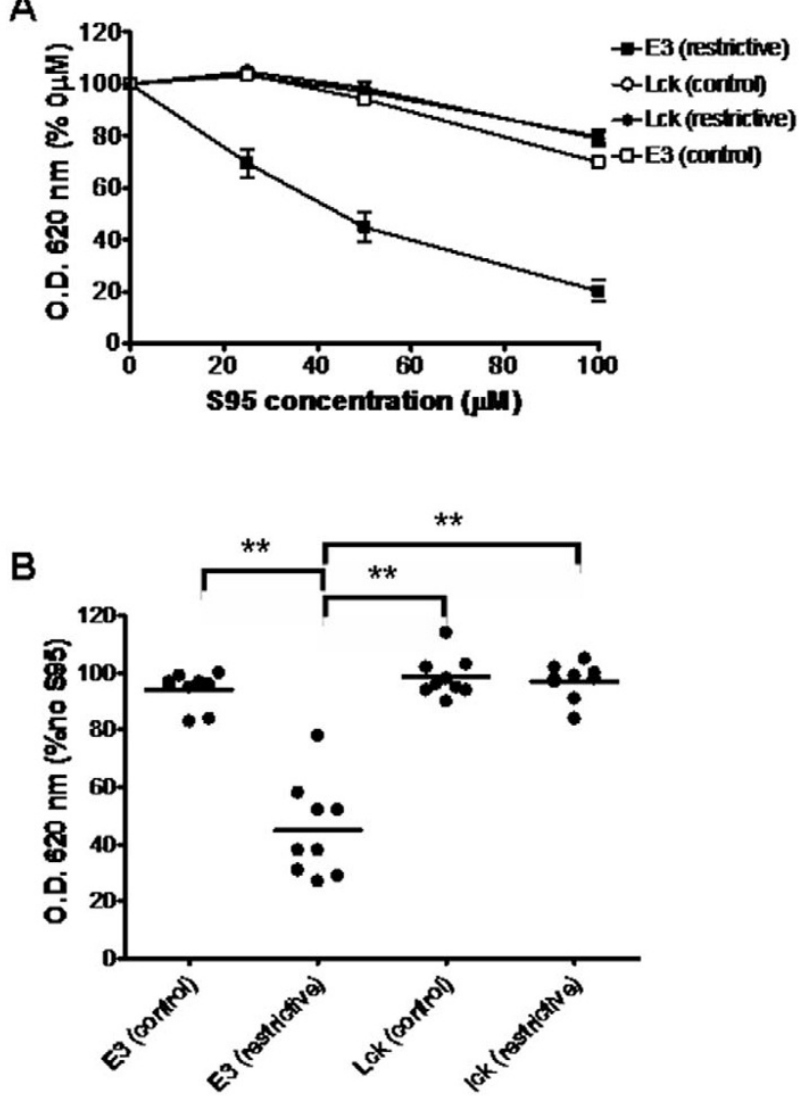

Figure 3

Effect of S9567 I I on the EGLN-dependent and Lckdependent three-hybrid systems. Yeast clones transformed with constructs encoding for AD-P564 and E3/DBVHL (E3) or AD-SH2 and Lck/DB-ITAM (Lck) were used to inoculate control media lacking Leu and Trp (control) or restrictive media (restrictive) lacking Leu, Trp, and His (in the case of E3 clones, restrictive media also lacked adenine). Duplicate cell cultures were grown in the presence of 0,25 , 50 or $100 \mu \mathrm{M}$ S9567II. The density of cultures was determined 16-24 hours after inoculation. A, The cell density (absorbance at $620 \mathrm{~nm}$ ) of each culture condition is represented as the percentage of density obtained for the cells grown in the absence of drug. The graph represents the average values of nine independent experiments. Error bars represent the S.E.M. B, the individual data values of density for the $50 \mu \mathrm{M}$ S9567II cultures in A are represented. Horizontal bars represent the mean. Statistically significant different mean values $(p<0.00 \mathrm{I})$ are indicated with asterisks.

not affect EGLN binding to substrate. Therefore, the effect of this drug on the reconstituted HIF system (figure 4) is likely due to its effect on EGLN activity rather than the EGLN3/HIF interaction. It should be noted however that these results do not exclude a potential effect of DMOG on VHL/hydroxylated-HIF interaction.
In addition to S956711 and DMOG, we also tested more general inhibitors, such as deferoxamine and cobalt chloride, that have been widely used to activate HIF $[8,14]$. EGLNs contain a tightly bound $\mathrm{Fe}^{+2}$ atom in the catalitic site that is required as a cofactor in the hydroxylation reaction. Consequently, iron sequestration by the chelating agent deferoxamine results in EGLN inhibition $[8,14]$. On the other hand, the mechanism of EGLN inhibition by cobalt chloride is controversial $[43,44]$. It might act by substitution of the iron from the enzyme catalytic site and/or interfere with ascorbate transport [44], which is required to prevent the oxidation of the catalytic site of the enzyme. In our assays, cobalt chloride had an important toxic effect on yeast and showed little specific inhibition of the EGLN system (data not shown), thus we did not study it in further detail. On the other hand, we were unable to detect any significant effect of deferoxamine on yeast growth at concentrations up to $1 \mathrm{mM}$ (data not shown). A possible explanation for the lack of effect of deferoxamine is that treatment might induce an adaptive response in yeast that increases their iron uptake [45]. In spite of their wide use as hypoxia mimetics, a recent report describes that cobalt and deferoxamine are ineffective inhibitors of EGLNs in vitro [43], which is in agreement with the lack of significant effect of these drugs in our system.

All together these results indicate that the combination of the control (Lck-dependent) and experimental (EGLNdependent) systems constitutes an efficient assay to identify small molecules that have a specific effect on EGLN activity, while revealing those that have unspecific or toxic effects. Importantly, the effect of S956711 and DMOG in our system was achieved at doses equivalent to those required for the inhibition of EGLNs in mammalian cells $[31,41]$.

\section{Discussion}

Herein we describe a yeast three-hybrid system that reconstitutes HIF regulation through proline hydroxylation and VHL interaction. This system accurately preserves EGLN substrate specificity, supporting its biochemical relevance. The system described in this work relies on the interaction between a 22 residue fragment derived from HIF $1 \alpha$ and the $\beta$-domain of VHL. The HIF1 $\alpha$ fragment can be replaced by the full length protein without affecting the performance of system, except for a slight reduction in the interaction strength (data not shown). However, we were unable to express full length VHL in yeast (data not shown), probably due to misfolding of its $\alpha$-domain in the absence of elongins B and C [46,47]. To circumvent this problem and since only the $\alpha$ domain of VHL interacts with elongin B [46], we generated a truncated VHL lacking this domain. This VHL form contains an intact $\beta$ domain [36] that, according to our data, is sufficient to 
A
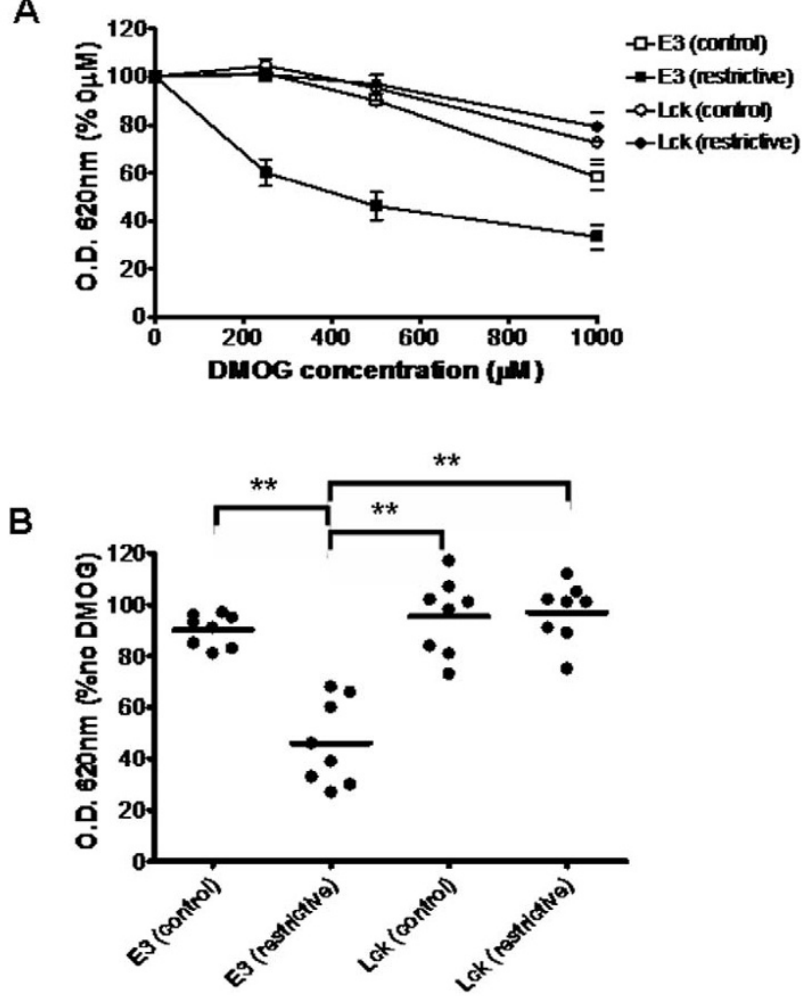

Figure 4

Effect of DMOG on the EGLN-dependent and Lckdependent three-hybrid systems. Yeast clones transformed with constructs encoding for AD-P564 and E3/DBVHL (E3) or AD-SH2 and Lck/DB-ITAM (Lck) were used to inoculate control media lacking Leu and Trp (control) or restrictive media (restrictive) lacking Leu, Trp, and His (in the case of E3, clones restrictive media also lacked adenine). Duplicate cell cultures were grown in the presence of 0,250 , 500 or $1000 \mu$ M DMOG. The density of cultures was determined 16-24 hours after inoculation. A, The cell density (absorbance at $620 \mathrm{~nm}$ ) of each culture condition is represented as the percentage of density obtained for the cells grown in the absence of drug. The graph represents the average values of eight independent experiments. Error bars represent the S.E.M. B, the individual data values of density for the $500 \mu$ M DMOG cultures in A are represented. Horizontal bars represent the mean. Statistically significant different mean values $(p<0.00 I)$ are indicated with asterisks.

bind HIF. Although the interaction of this truncated VHL construct with HIF is robust, we can not rule out that its affinity for HIF is different to that of the native VBC complex. In summary, this system is probably composed of the minimal functional units required to reconstitute HIF1 $\alpha$ regulation.

Given the relevance of hypoxia in several pathologies, the identification of small molecules with the ability to mod- ulate HIF activity has drawn much interest. In this regard, we have demonstrated that the three-hybrid assay described herein can be used to effectively identify small molecules that interfere with HIF regulation. Importantly, in contrast to current enzymatic assays, this system can be easily adapted to be performed on 96-well plates so it can be used for high-throughput screening of small molecules. In this regard, it should be noted that the direct determination of yeast growth provides a narrow linear range. Thus, in order to adapt this system to large high throughput screenings it would be much more convenient to determine $\beta$-galactosidase activity. Several yeast strains contain a LacZ gene under the control of the Gal4 promoter, thus $\beta$-galactosidase activity reflects the strength of the interaction between fusion proteins. In addition, the controls included in this work have proved useful in discriminating between genuine specific inhibitors of the EGLN-dependent system and molecules with a broad spectrum of targets such as cobalt chloride. Since the control (Lck-dependent assay) is based in exactly the same methodology than the test (EGLN-dependent assay), the combination of both systems constitutes a convenient assay for large screenings. Finally, comparison of the effects of the drug on the whole reconstituted system (three-hybrid) and on the direct interaction between EGLNs and HIFa (two-hybrid) could be helpful in the elucidation of the inhibition mechanism of candidate drugs (figure 5).

When considering the modulation of the HIF pathway, it is important to take into account that the relative contribution of each EGLN to HIF regulation seems to be different $[17,22,23]$ and probably cell-type specific [21]. Moreover, it is likely that different EGLN isoforms might have a specific set of target molecules $[24,25]$. Thus, the development of isoform-specific inhibitors is of great interest. However, since the catalitic site of the three EGLNs members is very similar, the identification of specific inhibitors based on analogues of the 2-OG is probably difficult [48]. On the other hand, our work [49] and that of others [50] have recently demonstrated that the different substrate specificity shown by each EGLN isoform relies on a defined substrate-binding surface relatively far from the catalitic site. Thus, it is theoretically possible to identify molecules that block the HIF-binding region in specific EGLN isoforms. In this regard, use of our system, in particular the direct interaction assay (figure 5 and reference[23]), could aid in the identification/characterization of such a molecule.

During the writing of this manuscript a paper was published describing a yeast two-hybrid system that, similarly to ours, reconstitutes HIF recognition by VHL [51]. However, an important practical difference between the two systems is that the one described by Bex and coworkers 
A
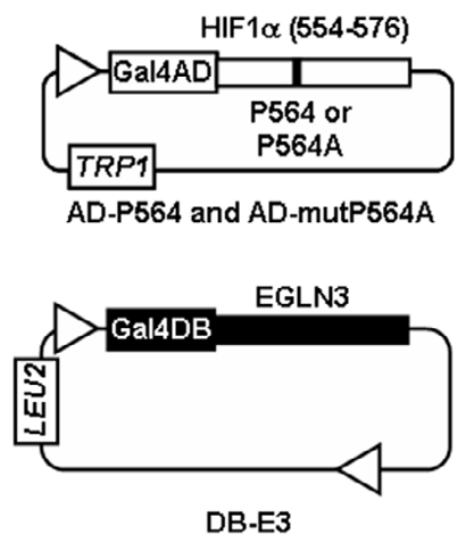

B

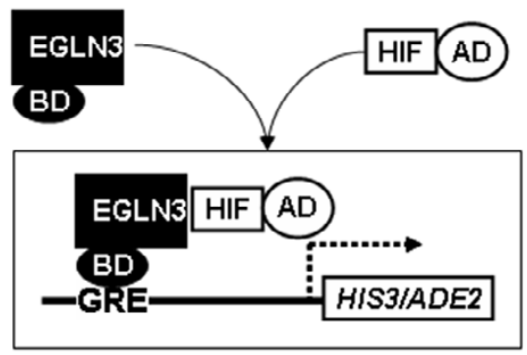

C

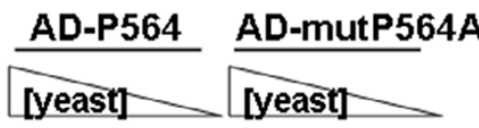

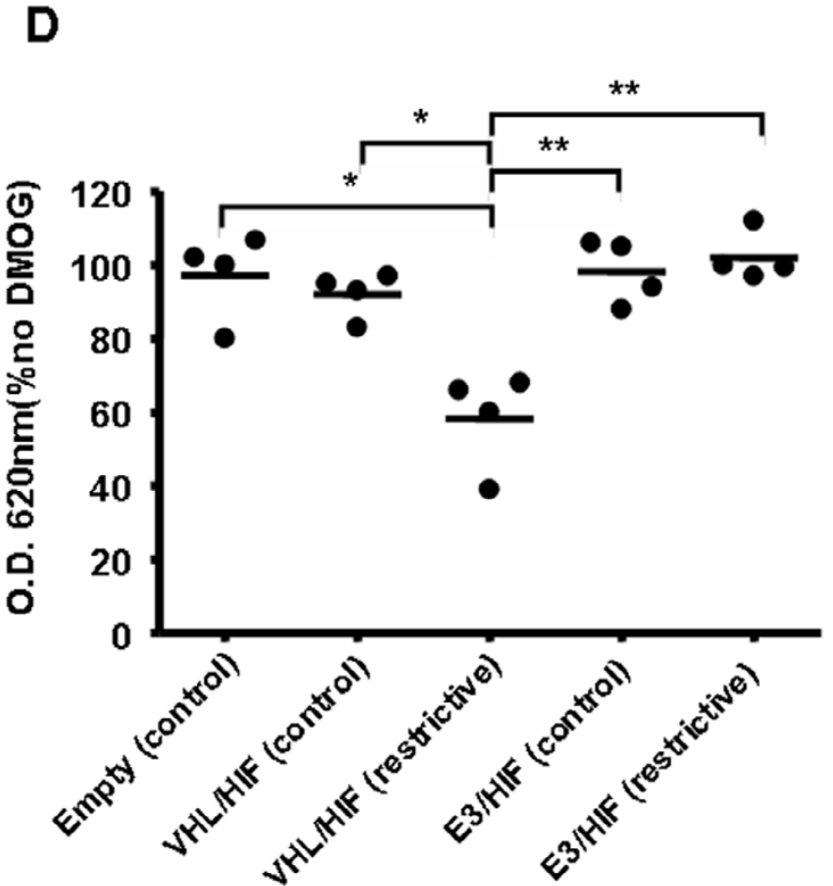

Figure 5

Effect of DMOG on the direct binding of EGLN to HIF. Schematic diagram representing the constructs (A) and the interaction between the different system components (B). C, Serial dilutions of clones transformed with the indicated constructs were grown on different stringency plates. The results shown are representative of at least three independent experiments. D, Yeast clones transformed with empty PGAD and pBridge plasmids (Empty) or with constructs encoding for ADP564 and E3/DB-VHL (VHL/HIF) or AD-P564 and DB-E3 (E3/HIF) were used to inoculate control media lacking Leu and TrP (control) or restrictive media (restrictive) lacking Leu, Trp, and His (in the case of E3 clones restrictive media also lacked adenine). Duplicate cell cultures were grown in the presence of 0 or $500 \mu M$ DMOG and the density of cultures was determined 16-24 hours after inoculation. The density values obtained for cultures treated with DMOG were normalized as percentage of the density obtained for cultures in the absence of drug. The graph represents the individual data values of density for each of the four independent experiments. Horizontal bars represent the mean. Statistically significant different mean values are indicated with one $(p<0.05)$ or two $(p<0.01)$ asterisks. Symbols, abbreviations and panel labels are as in figure I.

required the coexpression of elongins in order to obtain a functional full length VHL.

\section{Conclusion}

In summary, the results presented indicate that this system constitutes a simple yet powerful assay to study EGLN biochemistry in a cellular context without the interference of the endogenous HIF system. The system is particularly well suited to screen for modulators of the HIF regulatory machinery. Finally, since the specificity of the reaction cat- alyzed by the EGLNs is accurately preserved, this system can also be used to characterize novel EGLNs targets.

\section{Methods}

\section{Cells and reagents}

The S. cerevisiae AH109 strain, yeast growth media reagents YPD (yeast extract/peptone/dextrose), SD (synthetic defined) amino acids, X-gal (5-bromo-4-chloroindol-3-yl $\beta$-D-galactopyranoside) and plasmids (pGAD and pBridge) were from BD Biosciences, (Palo Alto, CA). 
Table I: Primers used for the generation of the constructs used in this study.

\begin{tabular}{lll}
\hline Gene & \multicolumn{2}{c}{ Primer } \\
\hline Syk & Fw & 5'GAATTCAACCACCTGCCCTTCTTT 3' \\
& Rv & 5'GAATTCTGCCGATTTTTTGACATGG 3' \\
VHL & Fw & 5'AGCTGAATTCCTGCGCTCGGTGAACTCGCGC3' \\
& Rv & 5'AGCTCTCGAGGGATCCTCAAGTATACACTGGCAGTGTGATATTG3' \\
FcERl $\gamma$ & $\mathrm{Fw}$ & 5'GAATTCCGACTGAAGATCCAAGTGCGAAAG 3' \\
& $\mathrm{Rv}$ & 5'GAATTCCTACTGTGGTGGTTTCTCATGCTTCAG 3' \\
Lck & $\mathrm{Fw}$ & 5'TGATCAGCGGCCGCCATGGGCTGTGGCTGCAGCTCACAC 3' \\
& $\mathrm{Rv}$ & 5'GCGGCCGCTGATCATCAAGGCTGAGGCTGGTACTGGCC 3' \\
EGLN3 & $\mathrm{Fw}$ & 5'AGCTAAGCTTACCATGAGATCTTCTAGAATGCCCCTGGGACACATCATGAGG 3' \\
& $\mathrm{Rv}$ & 5'GACTGAATTCTGATCAGTCGACGTCTTCAGTGAGGGCAGATTCAG 3' \\
& & \\
\hline
\end{tabular}

\section{Plasmid constructs}

Constructs expressing HIF1 $\alpha(554-576)$ and hSYK(11263) as Gal4AD fusion proteins were generated by PCR amplification (figure 5 and reference [23]) of the indicated coding region from human cDNA and cloned into the EcoRI/BamHI or EcoRI sites of pGAD-T7 plasmid (BD Biosciences) respectively. The Gal4DBD fusion proteins were generated by PCR amplification (table 1) of the indicated coding regions from the cloned full length sequence (VHL) or from human cDNA (FceRI $\gamma$ ). VHL (encoding for residues 63-157) and FceRI $\gamma$ (encoding for residues 4586) amplicons were then cloned into the XmaI/SalI or EcoRI sites respectively. Finally, the coding sequences for EGLN3 and LCK were PCR-amplified (table 1) and cloned into the BglII or NotI/BglII sites of pBRIDGE-VHL(63$157)$ or pBRIDGE-FceRI $\gamma(45-86)$ constructs respectively. LCK was amplified from human cDNA and EGLN-3 was amplified from its cloned cDNA, which was generously provided by Steven L. McKnight. For the two hybrid assays, the constructs expressing Gal4DBD fused to EGLN-3 were generated by cloning its coding sequence into the XmaI/SalI sites of the pBRIDGE plasmid (figure 5 and reference [23]).
In Table 2 are summed up all the constructs used in this study.

\section{Yeast transformation and plate interaction control assays} Freshly made competent yeast cells were transformed with $0.1 \mu \mathrm{g}$ or $0.3 \mu \mathrm{g}$ of GAL4AD or GAL4DBD constructs respectively by a modified version of the lithium acetate method (Gietz y col., 1992) and plated on minimal SD media plates in the absence of Leu and Trp for selection of transformants. Subsequently, an equal number of colonies from each transformation was transferred to saline solution $(0.9 \% \mathrm{NaCl})$ and subjected to serial dilutions. Aliquots of each cell suspension (typically $8 \mu \mathrm{l}$ ) were plated on culture media with different stringencies: (1) control plates lacking leucine/tryptophan (minimal stringency; no interaction between fusion proteins required for yeast growth); (2) restrictive plates lacking leucine, tryptophan and histidine (medium stringency; interaction required to support yeast growth); or (3) restrictive plates lacking leucine, tryptophan, histidine and adenine (maximal stringency; strong interaction required to support yeast growth).

Table 2: Plasmids used in this study.

\begin{tabular}{|c|c|c|c|c|c|c|}
\hline \multirow[b]{2}{*}{ NAME } & \multirow[b]{2}{*}{ VECTOR } & \multicolumn{3}{|c|}{ Expression from PADHI promoter } & \multicolumn{2}{|c|}{ Expression from PMET25 promoter } \\
\hline & & Protein & Fragment & MUTATIONS & Protein & Fragment \\
\hline AD-P564 & PGAD-T7 & GAL4 AD- HIF & $554-576$ & wt & & \\
\hline AD-mutP564A & PGAD-T7 & GAL4 AD- HIF & $554-576$ & P564A & & \\
\hline AD-L574A & PGAD-T7 & GAL4 AD- HIF & $554-576$ & L574A & & \\
\hline AD-L562R & pGAD-T7 & GAL4 AD- HIF & $554-576$ & L562R & & \\
\hline AD-P402 & PGAD-T7 & GAL4 AD- HIF & $392-414$ & wt & & \\
\hline AD-SH2 & PGAD-T7 & GAL4 AD- SYK SH2 & $11-236$ & wt & & \\
\hline DB-VHL & PBRIDGE & GAL4 DBD- VHL & $63-157$ & wt & & \\
\hline E3/DB-VHL & PBRIDGE & GAL4 DBD- VHL & $63-157$ & wt & EGLN3 & Full length \\
\hline $\mathrm{EI} / \mathrm{DB}-\mathrm{VHL}$ & pBRIDGE & GAL4 DBD- VHL & $63-157$ & wt & EGLNI & Full length \\
\hline DB-ITAM & pBRIDGE & GAL4 DBD- ITAM & $45-86$ & wt & & \\
\hline LCK/DB-ITAM & pBRIDGE & GAL4 DBD- ITAM & $45-86$ & wt & LCK & Full length \\
\hline DB-E3 & PBRIDGE & GAL4 DBD- EGLN3 & Full length & wt & & \\
\hline
\end{tabular}




\section{Liquid media yeast growth assays}

An equal number of fresh (up to two weeks old) yeast colonies were transferred to $6 \mathrm{ml}$ of minimal stringency liquid media and grown overnight at $250 \mathrm{rpm}$ and $30^{\circ} \mathrm{C}$. Yeast cells were subsequently pelleted, thoroughly washed with maximal stringency medium and resuspended in 1 $\mathrm{ml}$ of the appropriate liquid culture media at a fixed concentration as determined in pilot experiments (Additional file 2). Cultures were then grown at $250 \mathrm{rpm}$ and $30^{\circ} \mathrm{C}$ for the indicated times. Growth of cell cultures was measured as the increase in $620 \mathrm{~nm}$ absorbance of $150 \mu \mathrm{l}$ culture aliquots taken at initial $(\mathrm{t}=0)$ and the indicated times. For the drug assays, drugs were added to the culture media at $\mathrm{t}=0$.

\section{Statistical analysis of data}

Experimental data were analyzed with the Prism ${ }^{\mathrm{TM}}$ GraphPad (version 4.01) software. Data were analyzed by the analysis of variance test (ANOVA) followed by the Tukey's multiple comparison test.

\section{List of abbreviations}

HIF, hypoxia inducible factor; EGLN, EGL-nine Homolog; ODD, Oxygen-dependent Degradation Domain; AD, Gal4 activation domain; DB, Gal4 DNA binding domain; DMOG, dimethyloxaloylglycine.

\section{Authors' contributions}

MA carried out all the experiments shown in figures 1,2 , 3, 4, 5 and Additional file 1. AVV generated the HIF/VHL/ EGLN constructs for the two- and three-hybrid assays and did all the preliminary experiments for the hybrid assays. She also performed the experiments in Additional file 1. MOL and LFGF participated in the design and coordination of the study. LdP conceived the study, and participated in its design and coordination. He also performed the statistical analysis and wrote the manuscript. All authors read and approved the final manuscript.

\section{Additional material}

\section{Additional file 1}

Characterization of the EGLN substrate specificity in the yeast threehybrid system. Yeast cells were treated as indicated in figure 1. ADL574A and AD-L562R encode for the Gal-4 activation domain fused to HIF1 $\alpha$ derived peptides expanding residues 554-576 and containing L574A or L562R mutations respectively. Mutation L574A prevents HIF hydroxylation by EGLNs [39]. In agreement, expression of none of the EGLN isoforms was able to trigger DB-VHL binding to AD-L574A in spite of the presence of an intact proline 564 residue. In contrast, mutation of L562R specifically affected the recognition of the substrate by EGLN3, while EGLN1 was unaffected. These results are in agreement with previous reports that indicate a preference of EGLN1, but not EGLN3, for arginine at that particular position $[23,37]$. The result shown is representative of two independent experiments.

Click here for file

[http://www.biomedcentral.com/content/supplementary/1471-

2121-9-18-S1.png]

\section{Additional file 2}

kinetics of growth in liquid media. Yeast cells were transformed with constructs expressing AD-P564 and E3/DB-VHL or AD-SH2 and Lck/ DB-ITAM. After transformation, cells were selected on plates lacking Leu and Trp. Individual transformed clones were harvested and resuspended in liquid media. The initial concentration of each yeast culture was adjusted in preliminary experiments so that all cultures achieved logarithmic growth in overlapping time windows. The figure shows the absolute densities of each culture at different time points after inoculation. In our assay conditions, the different cultures shared a wide time window (between 16 to 30 hours of growth) in which they all were in the exponential phase of growth. A representative experiment, of at least three independent, is shown.

Click here for file

[http://www.biomedcentral.com/content/supplementary/14712121-9-18-S2.png]

\section{Acknowledgements}

We thank Diego Villar for critical reading of this manuscript and his valuable suggestions. This work was supported by grants from Ministerio de Ciencia y Tecnología (SAF2005-00I80 to L. P. and SAF 2007-60592 to M.O.L), from Red Cardiovascular (RECAVA to M.O.L.) and Comunidad Autónoma de Madrid (S-SAL-03II_2006 to L.P. and M.O.L.).

\section{References}

I. Brahimi-Horn MC, Chiche J, Pouyssegur J: Hypoxia signalling controls metabolic demand. Current Opinion in Cell Biology 2007. 19(2):223-229.

2. Fukuda R, Zhang H, Kim J, Shimoda L, Dang CV, Semenza GL: HIF-I Regulates Cytochrome Oxidase Subunits to Optimize Efficiency of Respiration in Hypoxic Cells. Cell 2007, I 29(I): I|I-I22.

3. Zhang H, Gao P, Fukuda R, Kumar G, Krishnamachary B, Zeller KI, Dang CV, Semenza GL: HIF-I Inhibits Mitochondrial Biogenesis and Cellular Respiration in VHL-Deficient Renal Cell Carcinoma by Repression of C-MYC Activity. Cancer Cell 2007. II(5):407-420.

4. Hickey MM, Simon MC, Gerald PS: Regulation of Angiogenesis by Hypoxia and Hypoxia-Inducible Factors. In Current Topics in Developmental Biology Volume 76. Academic Press; 2006:217-257. 
5. Semenza GL: Oxygen-dependent regulation of mitochondrial respiration by hypoxia-inducible factor I. Biochem J 2007, 405(I): I-9.

6. Wenger RH, Stiehl DP, Camenisch G: Integration of oxygen signaling at the consensus HRE. SCi STKE 2005, 306:re I2.

7. Ivan M, Haberberger T, Gervasi DC, Michelson KS, Gunzler V, Kondo K, Yang H, Sorokina I, Conaway RC, Conaway JW, Kaelin WG Jr.: Biochemical purification and pharmacological inhibition of a mammalian prolyl hydroxylase acting on hypoxia-inducible factor. PNAS 2002, 99(2I): 13459-13464.

8. Jaakkola P, Mole DR, Tian YM, Wilson MI, Gielbert J, Gaskell SJ, Kriegsheim A, Hebestreit HF, Mukherji M, Schofield C], Maxwell PH, Pugh CW, Ratcliffe PJ: Targeting of HIF-alpha to the von HippelLindau ubiquitylation complex by O2-regulated prolyl hydroxylation. Science 200I, 292(55 I6):468-472.

9. Lando D, Peet DJ, Whelan DA, Gorman JJ, Whitelaw ML: Asparagine hydroxylation of the HIF transactivation domain a hypoxic switch. Science 2002, 295(5556):858-86।.

10. Hewitson KS, McNeill LA, Riordan MV, Tian YM, Bullock AN, Welford RW, Elkins JM, Oldham NJ, Shoumo B, Gleadle JM, Ratcliffe PJ, Pugh CW, Schofield CJ: Hypoxia-inducible Factor (HIF) asparagine hydroxylase is identical to factor inhibiting HIF (FIH) and is related to the cupin structural family. J Biol Chem 2002, 277(29):2635I-26355.

II. Lando D, Peet DJ, Gorman JJ, Whelan DA, White MF, Bruick RK: $\mathrm{FIH}-\mathrm{I}$ is an asparaginyl hydroxylase enzyme that regulates the transcriptional activity of hypoxia-inducible factor. Genes Dev 2002, 16:1466-147|.

12. Taylor MS: Characterization and comparative analysis of the EGLN gene family. Gene 200I, 275(I):I25-I32.

13. Masson N, Willam C, Maxwell PH, Pugh CW, Ratcliffe PJ: Independent function of two destruction domains in hypoxia-inducible factor-alpha chains activated by prolyl hydroxylation. $E M B O$ J 200I, 20(I8):5197-5206.

14. Ivan M, Kondo K, Yang H, Kim W, Valiando J, Ohh M, Salic A, Asara JM, Lane WS, Kaelin WG Jr.: HIFalpha targeted for VHL-mediated destruction by proline hydroxylation: implications for O2 sensing. Science 200I, 292(55 I 6):464-468.

15. Maxwell PH, Wiesener MS, Chang GW, Clifford SC, Vaux EC, Cockman ME, Wykoff CC, Pugh CW, Maher ER, Ratcliffe PJ: The tumour suppressor protein VHL targets hypoxia-inducible factors for oxygen-dependent proteolysis. Nature 1999 399(6733):27I-275.

16. Ehrismann D, Flashman E, Genn DN, Mathioudakis N, Hewitson KS, Ratcliffe PJ, Schofield C]: Studies on the activity of the hypoxiainducible-factor hydroxylases using an oxygen consumption assay. Biochem J 2007, 40 I (I):227-234.

17. Koivunen P, Hirsila M, Kivirikko KI, Myllyharju J: The length of peptide substrates has a marked effect on hydroxylation by the HIF prolyl 4-hydroxylases. The Journal of Biological Chemistry 2006 28I:287| 2-28720

18. Stolze IP, Tian YM, Appelhoff RJ, Turley H, Wykoff CC, Gleadle JM, Ratcliffe PJ: Genetic Analysis of the Role of the Asparaginy Hydroxylase Factor Inhibiting Hypoxia-inducible Factor (HIF) in Regulating HIF Transcriptional Target Genes. The Journal of Biological Chemistry 2004, 279(4I):42719-42725.

19. Bruick RK, McKnight SL: A conserved family of prolyl-4-hydroxylases that modify HIF. Science 200I, 294(5545): I337-I340.

20. Epstein AC, Gleadle JM, McNeill LA, Hewitson KS, O'Rourke J, Mole DR, Mukherji M, Metzen E, Wilson MI, Dhanda A, Tian YM, Masson N, Hamilton DL, Jaakkola P, Barstead R, Hodgkin J, Maxwell PH, Pugh CW, Schofield CJ, Ratcliffe PJ: C. elegans EGL-9 and Mammalian Homologs Define a Family of Dioxygenases that Regulate HIF by Prolyl Hydroxylation. Cell 200I, I 07( I):43-54

21. Appelhoff RJ, Tian YM, Raval RR, Turley H, Harris AL, Pugh CW, Ratcliffe PJ, Gleadle JM: Differential function of the prolyl hydroxylases PHDI, PHD2 and PHD3 in the regulation of the hypoxia inducible factor. The Journal of Biological Chemistry 2004, 279(37):38458-38465.

22. Hirsilä M, Koivunen P, Günzler V, Kivirikko KI, Myllyharju J: Characterization of the Human Prolyl 4-Hydroxylases That Modify the Hypoxia-Inducible Factor HIF. J Biol Chem 2003, 278(33):30772-30780.

23. Landazuri MO, Vara-Vega A, Viton M, Cuevas Y, del Peso L: Analysis of HIF-prolyl hydroxylases binding to substrates. Biochemical and Biophysical Research Communications 2006, 35 I (2):3 I3-320.
24. Cummins EP, Berra E, Comerford KM, Ginouves A, Fitzgerald KT, Seeballuck F, Godson C, Nielsen JE, Moynagh P, Pouyssegur J, Taylor $\mathrm{CT}$ : Prolyl hydroxylase-I negatively regulates IkappaB kinase-beta, giving insight into hypoxia-induced NFkappaB activity. PNAS 2006, $103(48)$ : I8I54-I8I59.

25. Koditz J, Nesper J, Wottawa M, Stiehl DP, Camenisch G, Franke C, Myllyharju J, Wenger RH, Katschinski DM: Oxygen-dependent ATF-4 stability is mediated by the PHD3 oxygen sensor. Blood 2007, I I 0(10):3610-36I7.

26. Bartrons R, Caro J: Hypoxia, glucose metabolism and the Warburg's effect. Journal of Bioenergetics and Biomembranes 2007, 39(3):223-229.

27. Calzada MJ, Del Peso L: Hypoxia-inducible factors and cancer. Clinical and Translational Oncology 2007, 9(5):278-289.

28. Bernhardt WM, Warnecke C, Willam C Tanaka T, Wiesener MS, Eckardt KU, Helmut Sies and Bernhard B: Organ Protection by Hypoxia and Hypoxia-Inducible Factors. In Methods in Enzymology Volume 435. Academic Press; 2007:219, 221-245.

29. Cai Z, Zhong H, Bosch-Marce M, Fox-Talbot K, Wang L, Wei C, Trush MA, Semenza GL: Complete loss of ischaemic preconditioning-induced cardioprotection in mice with partialdeficiency of HIF-I \{alpha\}. Cardiovasc Res 2007:cvm035.

30. Melillo G, Helmut Sies and Bernhard B: Hypoxia-Inducible Factor I Inhibitors. In Methods in Enzymology Volume 435. Academic Press; 2007:385-402.

31. Warnecke C, Griethe W, Weidemann A, Jürgensen JS, Willam C Bachmann S, Ivashchenko Y, Wagner I, Frei U, Wiesener M, Eckardt $\mathrm{KU}$ : Activation of the hypoxia-inducible factor pathway and stimulation of angiogenesis by application of prolyl hydroxylase inhibitors. The FASEB Journal 2003, I 7(9): I I 86- I I 88.

32. Hewitson KS, Schofield CJ, Ratcliffe PJ, Helmut Sies and Bernhard B Hypoxia-Inducible Factor Prolyl-Hydroxylase: Purification and Assays of PHD2. In Methods in Enzymology Volume 435. Edited by: Br HSB. Academic Press; 2007:25-42.

33. Wirthner R, Balamurugan K, Stiehl DP, Barth S, Spielmann P, Oehme F, Flamme I, Katschinski DM, Wenger RH, Camenisch G, Helmut Sies and Bernhard B: Determination and Modulation of Prolyl-4Hydroxylase Domain Oxygen Sensor Activity. In Methods in Enzymology Volume 435. Academic Press; 2007:43-60.

34. Arquier N, Vigne P, Duplan E, Hsu T, Therond PP, Frelin C, D'Angelo G: Analysis of the hypoxia-sensing pathway in Drosophila melanogaster. Biochem / 2006, 393(2):47| -480.

35. Chan DA, Sutphin PD, Denko NC, Giaccia A): Role of Prolyl Hydroxylation in Oncogenically Stabilized Hypoxia-inducible Factor-Ialpha. The Journal of Biological Chemistry 2002, 277(42):40 I I 2-40 I I7

36. Hon WC, Wilson MI, Harlos K, Claridge TDW, Schofield CJ, Pugh CW, Maxwell PH, Ratcliffe PJ, Stuart DI, Jones EY: Structural basis for the recognition of hydroxyproline in HIF-Ialpha by pVHL. Nature 2002, 417(6892):975-978.

37. Li D, Hirsila M, Koivunen P, Brenner MC, Xu L, Yang C, Kivirikko KI, Myllyharju J: Many amino acid substitutions in a HIF-Ialphalike peptide cause only minor changes in its hydroxylation by the HIF prolyl 4-hydroxylases: Substitution of 3,4-dehydroproline or azetidine-2-carboxylic acid for the proline leads to a high rate of uncoupled 2-oxoglutarate decarboxylation. The Journal of Biological Chemistry 2004, 279:5505 I-55059.

38. Huang J, Zhao Q, Mooney SM, Lee FS: Sequence Determinants in Hypoxia-inducible Factor-I a for Hydroxylation by the Prolyl Hydroxylases PHDI, PHD2, and PHD3. J Biol Chem 2002, 277(42):39792-39800.

39. Kageyama Y, Koshiji M, To KKW, Tian YM, Ratcliffe PJ, Huang LE: Leu-574 of human HIF-Ialpha is a molecular determinant of prolyl hydroxylation. FASEB ] 2004, I 8(9): 1028-1030.

40. Osborne MA, Dalton S, Kochan JP: The yeast tribrid system-genetic detection of trans-phosphorylated ITAM-SH2-interactions. Biotechnology 1995, I3(13): 1474-1478.

4I. Asikainen TM, Ahmad A, Schneider BK, Ho WB, Arend M, Brenner $M$, Gunzler V, White CW: Stimulation of HIF-Ialpha, HIF2alpha, and VEGF by prolyl 4-hydroxylase inhibition in human lung endothelial and epithelial cells. Free Radical Biology and Medicine 2005, 38(8): 1002-10I3.

42. Cunliffe CJ, Franklin TJ, Hales NJ, Hill GB: Novel inhibitors of prolyl 4-hydroxylase. 3. Inhibition by the substrate analogue $\mathrm{N}$ oxaloglycine and its derivatives. Journal of Medicinal Chemistry 1992, 35(14):2652-2658. 
43. Hirsila M, Koivunen $\mathrm{P}, \mathrm{Xu}$ L, Seeley T, Kivirikko KI, Myllyharju J: Effect of desferrioxamine and metals on the hydroxylases in the oxygen sensing pathway. FASEB J 2005, 19(10): / 308-13 I0.

44. Salnikow K, Donald SP, Bruick RK, Zhitkovich A, Phang JM, Kasprzak KS: Depletion of Intracellular Ascorbate by the Carcinogenic Metals Nickel and Cobalt Results in the Induction of Hypoxic Stress. The Journal of Biological Chemistry 2004, 279(39):40337-40344.

45. Yun CW, Ferea T, Rashford J, Ardon O, Brown PO, Botstein D, Kaplan J, Philpott CC: Desferrioxamine-mediated Iron Uptake in Saccharomyces cerevisiae. EVIDENCE FOR TWO PATHWAYS OF IRON UPTAKE. The Journal of Biological Chemistry 2000, 275(14): 10709-107|5.

46. Stebbins CE, Kaelin WG Jr., Pavletich NP: Structure of the VHLElonginC-ElonginB complex: implications for VHL tumor suppressor function. Science 1999, 284(54I3):455-46I.

47. Tan S: A Modular Polycistronic Expression System for Overexpressing Protein Complexes in Escherichia coli. Protein Expression and Purification 200I, 2 I (I):224-234.

48. McDonough MA, Li V, Flashman E, Chowdhury R, Mohr C, Lienard BMR, Zondlo J, Oldham NJ, Clifton IJ, Lewis J, McNeill LA, Kurzeja RJM, Hewitson KS, Yang E, Jordan S, Syed RS, Schofield CJ: Cellular oxygen sensing: Crystal structure of hypoxia-inducible facto prolyl hydroxylase (PHD2). PNAS 2006, 103(26):98|4-98।9.

49. Villar D, Vara-vega A, LandÃ izuri MO, DelÂ peso L: Identification of a region on hypoxia-inducible-factor prolyl 4-hydroxylases that determines their specificity for the oxygen degradation domains. Biochem J 2007, 408(2):23I-240.

50. Flashman E, Bagg EAL, Chowdhury R, Mecinovic J, Loenarz C McDonough MA, Hewitson KS, Schofield CJ: Kinetic rationale for selectivity towards $\mathrm{N}$ - and $\mathrm{C}$-terminal oxygen dependent degradation domain substrates mediated by a loop region of HIF prolyl hydroxylases. The Journal of Biological Chemistry 2007:M7074 I I 200.

5I. Bex C, Knauth K, Dambacher S, Buchberger A: A yeast two-hybrid system reconstituting substrate recognition of the von Hippel-Lindau tumor suppressor protein. Nucl Acids Res 2007, 35(2I): el 42.

Publish with Bio Med Central and every scientist can read your work free of charge

"BioMed Central will be the most significant development for disseminating the results of biomedical research in our lifetime. "

Sir Paul Nurse, Cancer Research UK

Your research papers will be:

- available free of charge to the entire biomedical community

- peer reviewed and published immediately upon acceptance

- cited in PubMed and archived on PubMed Central

- yours - you keep the copyright
BioMedcentral 